\title{
The Inverse Gravity Inflationary Theory of Cosmology
}

\author{
Edward A. Walker \\ Mathematics Department, Florida Memorial University, Miami Gardens, FL, USA \\ Email: dwrdwalker@yahoo.com
}

How to cite this paper: Walker, E.A. (2016) The Inverse Gravity Inflationary Theory of Cosmology. Journal of Modern Physics, 7, 1762-1776.

http://dx.doi.org/10.4236/jmp.2016.713158

Received: August 12, 2016

Accepted: September 25, 2016

Published: September 28, 2016

Copyright $\odot 2016$ by author and Scientific Research Publishing Inc. This work is licensed under the Creative Commons Attribution International License (CC BY 4.0).

http://creativecommons.org/licenses/by/4.0/ (c) (i) Open Access

\begin{abstract}
Cosmological expansion or inflation is mathematically described by the theoretical notion of inverse gravity whose variations are parameterized by a factor that is a function of the distance to which cosmological expansion takes prominence over gravity. This assertion is referred to as the inverse gravity inflationary assertion. Thus, a correction to Newtonian gravitational force is introduced where a parameterized inverse gravity force term is incorporated into the classical Newtonian gravitational force equation where the inverse force term is negligible for distances less than the distance to which cosmological expansion takes prominence over gravity. Conversely, at distances greater than the distance to which cosmological expansion takes prominence over gravity. The inverse gravity term is shown to be dominant generating universal inflation. Gravitational potential energy is thence defined by the integral of the difference (or subtraction) between the conventional Newtonian gravitational force term and the inverse gravity term with respect to radius $(r)$ which allows the formulation, incorporation, and mathematical description to and of gravitational redshift, the Walker-Robertson scale factor, the Robinson-Walker metric, the Klein-Gordon lagrangian, and dark energy and its relationship to the energy of the big bang in terms of the Inverse gravity inflationary assertion. Moreover, the dynamic pressure of the expansion of a cosmological fluid in a homogeneous isotropic universe is mathematically described in terms of the inverse gravity inflationary assertion using the stress-energy tensor for a perfect fluid. Lastly, Einstein's field equations for the description of an isotropic and homogeneous universe are derived incorporating the mathematics of the inverse gravity inflationary assertion to fully show that the theoretical concept is potentially interwoven into the cosmological structure of the universe.
\end{abstract}

\section{Keywords}

Isotropic and Homogeneous Universe, Inverse Gravity, Cosmological Inflation, 
Gravitational Redshift, Robertson-Walker Scale Factor, Klein-Gordon Lagrangian, Dark Energy, Stress-Energy Tensor, Friedman-Walker-Robertson Metric, Photon

\section{Introduction}

The theoretical notion that cosmological expansion or universal inflation occurs due to inverse variations in gravitational force whose rate of change is regulated by a limiting factor or parameter is introduced. Thus, it is asserted that cosmological expansion or inflation is an inherent property of nature mathematically described by the difference between conventional Newtonian gravitational force and its inverse term which is multiplied by an inflationary parameter which regulates its rate of change. The inflationary parameter multiplied by the inverse term of Newtonian gravitational force is determined by (and is a function of) an astronomical distance to which cosmological expansion over takes gravitational force on a cosmic scale. The establishment of the core concept of the inverse gravity inflationary assertion aforementioned is the foundation to describing the universe in terms of the new assertion. Thus, the aim of this paper is the incorporation of the inverse gravity inflationary assertion (IGIA) into proven and established mathematics describing cosmological inflation.

A more detailed introduction is that this paper formulates the correction to the Newtonian gravitational force equation incorporating an inverse gravity term and its conditions. This permits the derivation of gravitational potential energy in terms of the IGIA. Resultantly, the relationship between gravitational potential energy, dark energy, gravitational redshift, the Klein-Gordon Lagrangian, the energy of the big bang, the Robertson-Walker scale factor, and the Friedman-Walker-Robertson metric in terms of the inverse gravity inflationary assertion (IGIA) is formulated and defined. The IGIA is then applied to the stress-energy tensor for describing the dynamic pressure of an expanding cosmological fluid in a homogeneous and isotropic universe. Lastly, the IGIA is applied to Einstein's field equations for the description of a spherically homogeneous isotropic universe which establishes the inverse gravity inflationary assertion. This will elucidate how the IGIA is interwoven into the cosmological structure of the universe.

\section{The Correction to the Newtonian Gravitational Force Equation and IGIA Inflationary Parameter}

To begin the heuristic derivation, mass values $m_{1}$ and $m_{2}$ are the combined masses of cosmological bodies (such as galaxies) evenly dispersed over an isotropic and homogeneous universe and $G$ is the gravitational constant. The terms of gravitational force which are a function of radius $r$ are given such that [1]:

$$
F_{g}(r)=\frac{G m_{1} m_{2}}{r^{2}}, F_{g}^{\prime}(r)=B_{0}\left[\frac{r^{2}}{G m_{1} m_{2}}\right]
$$

where $F_{g}(r)$ is the classical expression of Newtonian gravitational force and $F_{g}^{\prime}(r)$ 
is the inverse term of Newtonian gravitational force, constant $B_{0}$ is the inflationary factor or parameter. Inflationary factor $B_{0}$ is stated such that:

$$
B_{0}=\frac{1}{r_{0}^{2}} ; 1>B_{0}>0 ; r_{0}>1
$$

The constant $r_{0}$ is the astronomical distance to which cosmological expansion takes prominence over gravity. The inverse term of gravity $F_{g}^{\prime}(r)$ can be re-expressed in terms of distance $r_{0}$ such that:

$$
F_{g}^{\prime}(r)=\left[\frac{1}{r_{0}^{2}}\right]\left[\frac{r^{2}}{G m_{1} m_{2}}\right]
$$

The total gravitational force $F_{T}(r)$ or the Newtonian correction is stated as the difference between force values $F_{g}^{\prime}(r)$ and $F_{g}(r)$ such that:

$$
F_{T}(r)=F_{g}^{\prime}(r)-F_{g}(r)
$$

Therefore,

$$
F_{T}(r)=\left[\frac{1}{r_{0}^{2}}\right]\left[\frac{r^{2}}{G m_{1} m_{2}}\right]-\frac{G m_{1} m_{2}}{r^{2}}
$$

The direction (+ or -) of the value of total force $F_{T}(r)$ has relationships defined by the inequalities of radius $r$ and distance $r_{0}$ given by the conditions below.

$$
\begin{aligned}
& \text { For } r>r_{0} ;+F_{T}(r) \\
& \text { For } r<r_{0} ;-F_{T}(r)
\end{aligned}
$$

Condition (1.05) describes cosmological expansion $+F_{T}(r)$ away from the gravitational force center or gravitational source for distances $r>r_{0}$. Conversely, for condition (1.06), inverse gravity term $F_{g}^{\prime}(r)$ in total force $F_{T}(r)$ is negligible at distance $r<r_{0}$ causing force direction $-F_{T}(r)$ toward the center of gravitational force.

The value of the cosmological parameter of distance $r_{0}$ is determined where total force value $F_{T}(r)$ equals zero and radius $r$ equals cosmological parameter $r_{0} \quad\left(r=r_{0}\right.$ and $\left.F_{T}\left(r_{0}\right)=0\right)$. Furthermore, this implies that for the condition of $F_{T}\left(r_{0}\right)=0$, the force terms $F_{g}^{\prime}\left(r_{0}\right)$ and $F_{g}\left(r_{0}\right)$ in total force $F_{T}\left(r_{0}\right)$ are equal $\left(F_{g}^{\prime}\left(r_{0}\right)=F_{g}\left(r_{0}\right)\right)$. Therefore, total force $F_{T}\left(r_{0}\right)$ can be presented such that:

$$
F_{T}\left(r_{0}\right)=\left[\frac{1}{r_{0}^{2}}\right]\left[\frac{\left(r_{0}\right)^{2}}{G m_{1} m_{2}}\right]-\frac{G m_{1} m_{2}}{\left(r_{0}\right)^{2}}=0
$$

This reduces to:

$$
\left[\frac{1}{G m_{1} m_{2}}\right]-\frac{G m_{1} m_{2}}{\left(r_{0}\right)^{2}}=0
$$

Solving Equation (1.08) for the cosmological parameter of distance $r_{0}$ gives a value such that:

$$
r_{0}=G m_{1} m_{2}
$$

Conclusively Equation (1.09) above, gives the value of distance $r_{0}$ to which cosmo- 
logical expansion takes prominence over gravity. In describing an isotropic and Homogeneous spherical universe, mass values $m_{1}$ and $m_{2}$ will be evenly (and uniformly) distributed about the spherical volume. Mass value $m_{u}$ denotes the total mass of the universe, therefore the dispersion of cosmological mass $m_{u}$ will be described via the function $g(m, \theta, \phi)$ at mass variable $m$ and the spherical coordinates at $\theta$ and $\phi$ [2]. Resultantly, mass $m_{\mathrm{u}}^{\prime}$ (corresponding to each mass value of $m_{1}$ and $m_{2}$ ) which represents a portion of cosmological mass dispersed about the sphere is set equal to function $g(m, \theta, \phi)$ as shown below.

$$
m_{\mathrm{u}}^{\prime}=g(m, \theta, \phi)
$$

Function $g(m, \theta, \phi)$ takes on a value of [2]:

$$
g(m, \theta, \phi)=\left[(m \cos \theta \sin \phi)^{2}+(m \sin \theta \sin \phi)^{2}+(m \cos \phi)^{2}\right]^{1 / 2}
$$

The gravitational interaction of symmetric portions of cosmological mass $m_{u}^{\prime}$ and $m_{\mathrm{u}}^{\prime}$ separated by distance $r_{0}$ is stated as the product between the two mass values such that:

$$
m_{\Perp}^{\prime} m_{\Perp}^{\prime}=(g(m, \theta, \phi))(g(m, \theta, \phi))=(g(m, \theta, \phi))^{2}
$$

Thus, the continuous sums (or integration) of gravitational mass interaction $m_{\mathrm{u}}^{\prime} m_{\mathrm{u}}^{\prime}$ to whom are located on opposite sides of distance $r_{0}$ is taken to a value $\pi$ and to the value of the mass of the universe $m_{u}$. Thus, the gravitational interaction of mass values $m_{1} m_{2}$ in Equation (1.09) equals the triple integral shown below [2].

$$
m_{1} m_{2}=\int_{0}^{m_{u}} \int_{0}^{\pi} \int_{0}^{\pi} m\left(m_{\mathrm{u}}^{\prime} m_{\mathrm{u}}^{\prime}\right) \mathrm{d} m \mathrm{~d} \theta \mathrm{d} \phi
$$

As the continuous sums of the integrals in Equation (1.13) progress in concert with angles $\theta$ and $\phi$ and sum up to a value $\pi$, the interacting mass values of $m_{1}$ and $m_{2}$ on opposite sides of distance $r_{0}$ sum up in concert with variable mass $m$ to encompass both halves of the spherical volume, giving the gravitational interaction of the entire spherical volume. Thus, the integration of (1.13) gives the gravitational mass interaction of the entire spherical volume. Therefore substituting the value of Equation (1.13) into (1.09) gives the proper mathematical description of distance $r_{0}$ shown below.

$$
r_{0}=G\left[\int_{0}^{m_{u}} \int_{0}^{\pi} \int_{0}^{\pi} m\left(m_{\amalg}^{\prime} m_{\Perp}^{\prime}\right) \mathrm{d} m \mathrm{~d} \theta \mathrm{d} \phi\right]
$$

The aim and scope of this paper is to introduce the notion and mathematics of the Inverse gravity inflationary assertion, thus we leave the calculation and value of Equation (1.14) as an exercise to the scientific community based on data obtained (The value of universal mass $m_{u}$ ) by astronomical observations.

\section{The IGIA Mathematical Integration into Established Fundamental Concepts in Cosmology}

This section applies the mathematical concept of the inverse gravity assertion to gravitational potential energy, gravitational Redshift, the Robertson-Walker scale factor, 
Friedman-Walker-Robertson metric, the Klein-Gordon lagrangian, dark energy, and the energy of the big bang. Gravitational potential energy $U_{T}(r)$ describing the energy of inflation in terms of the IGIA is equal to the conventional integral of total force $F_{T}(r)$ (Equation (1.04)) with respect to radius $r$ (for the condition of $r>r_{0}$ ) as shown below [1].

$$
U_{T}(r)=\int F_{T}(r) \mathrm{d} r
$$

Thus after evaluating the integral above, one obtains a value of potential energy $U_{T}(r)$ such that:

$$
U_{T}(r)=\left[\frac{1}{r_{0}^{2}}\right]\left[\frac{r^{3}}{3 G m_{1} m_{2}}\right]+\frac{G m_{1} m_{2}}{r}
$$

As a photon propagates across the expanding cosmological expanse, its energy is affected by the gravitational potential energy $U_{T}(r)$. Thus, photonic energy $E$ is set equal to potential energy $U_{T}(r)$ in terms of the IGIA; this equivalence is displayed below [1].

$$
E=p c=\frac{h c}{\lambda_{g}}=U_{T}(r)
$$

where $\lambda_{g}$ is the photon's wavelength influenced by potential energy $U_{T}(r)$, the photonic energy affected by the potential energy field of $U_{T}(r)$ can be expressed such that $[1]$ :

$$
\frac{h c}{\lambda_{g}}=\left[\frac{1}{r_{0}^{2}}\right]\left[\frac{r^{3}}{3 G m_{1} m_{2}}\right]+\frac{G m_{1} m_{2}}{r}
$$

Resultantly, wavelength $\lambda_{g}$ affected by the potential energy field of $U_{T}(r)$ of the IGIA has a value expressed as [1]:

$$
\lambda_{g}=h c\left[\left[\frac{1}{r_{0}^{2}}\right]\left[\frac{r^{3}}{3 G m_{1} m_{2}}\right]+\frac{G m_{1} m_{2}}{r}\right]^{-1}
$$

Energy $E_{0}$ is the initial energy value $\left(E_{0}=\left(h c / \lambda_{0}\right)\right)$ of the emitted photon prior to it traversing through a region of space-time under the influence of gravitational potential energy $U_{T}(r)$ in terms of the IGIA, thus redshift $z$ is given such that [3]:

$$
Z=\frac{U_{T}(r)-E_{0}}{E_{0}}=\frac{\left(\frac{h c}{\lambda_{g}}\right)-E_{0}}{E_{0}} \equiv \frac{\lambda_{0}}{\lambda_{g}}-1
$$

This reduces to [3]:

$$
z=\frac{1}{E_{0}}\left(\frac{h c}{\lambda_{g}}\right)-1 \equiv \frac{\lambda_{0}}{\lambda_{g}}-1
$$

Red shift value $z$ can then be expressed in terms of the IGIA such that:

$$
z=\frac{1}{E_{0}}\left[\left[\frac{1}{r_{0}^{2}}\right]\left[\frac{r^{3}}{3 G m_{1} m_{2}}\right]+\frac{G m_{1} m_{2}}{r}\right]-1
$$


Thus, the photonic energy value $h c / \lambda_{g}$ is substituted by the value of potential energy $U_{T}(r)$ in Equation (2.06) giving the value of redshift $z$ in Equation (2.07). Observe the expression below where $a_{0}$ is the scale factor of the universe as it is presently and $a\left(t_{e m}\right)$ is the scale factor at the emission time $t_{e m}$ of the photon (or a scale factor of the universe as it was in the past as some authors state it) [4].

$$
1+z=\frac{a_{0}}{a\left(t_{e m}\right)}
$$

Substituting the value of redshift $z$ of Equation (2.07) into (2.08) above gives [4]:

$$
1+\left(\left(\frac{1}{E_{0}}\left[\left[\frac{1}{r_{0}^{2}}\right]\left[\frac{r^{3}}{3 G m_{1} m_{2}}\right]+\frac{G m_{1} m_{2}}{r}\right]\right)-1\right)=\frac{a_{0}}{a\left(t_{e m}\right)}
$$

This reduces to:

$$
\frac{1}{E_{0}}\left[\left[\frac{1}{r_{0}^{2}}\right]\left[\frac{r^{3}}{3 G m_{1} m_{2}}\right]+\frac{G m_{1} m_{2}}{r}\right]=\frac{a_{0}}{a\left(t_{e m}\right)}
$$

The value of the scale factor at the time of the emitted photon $a\left(t_{e m}\right)$ is given such that $[4]$ :

$$
a\left(t_{e m}\right)=a_{0}\left[\frac{1}{E_{0}}\left[\left[\frac{1}{r_{0}^{2}}\right]\left[\frac{r^{3}}{3 G m_{1} m_{2}}\right]+\frac{G m_{1} m_{2}}{r}\right]\right]^{-1}
$$

where Equation (2.11) is of the form $a(t)=1 /(1+z)$ [4] which implies that $a_{0}$ equals $1\left(a_{0}=1\right)$. Equation (2.11) adequately shows the relationship of the Robertson-Walker scale factors and gravitational redshift to the IGIA. At this juncture, the IGIA connection to Friedman-Lemaitre-Walker-Robertson metric $\mathrm{d} \Sigma^{2}$ is shown below [3].

$$
\mathrm{d} \Sigma^{2}=-\mathrm{d} t^{2}+a^{2}(t)\left[\frac{\mathrm{d} r^{2}}{1-k r^{2}}+r^{2} \mathrm{~d} \theta^{2}+r^{2} \sin ^{2} \theta \mathrm{d} \phi^{2}\right]
$$

The scale factor $a(t)$ in Equation (2.12) above is set equal to the scale factor $a\left(t_{e m}\right)$ of Equation (2.11) $\left(a(t)=a\left(t_{e m}\right)\right)$ giving Equation (2.12) such that (note: for our purposes $\left.t=t_{e m}\right)[3]$ :

$$
\mathrm{d} \Sigma^{2}=-\mathrm{d} t^{2}+a^{2}\left(t_{e m}\right)\left[\frac{\mathrm{d} r^{2}}{1-k r^{2}}+r^{2} \mathrm{~d} \theta^{2}+r^{2} \sin ^{2} \theta \mathrm{d} \phi^{2}\right]
$$

Substituting the value of scale factor $a\left(t_{e m}\right)$ Equation (2.11) into Equation (2.13) above gives the Friedman-Walker-Robertson metric in terms of the IGIA such that:

$$
\begin{aligned}
\mathrm{d} \Sigma^{2} & =-\mathrm{d} t^{2} \\
& +\left[a_{0}\left[\frac{1}{E_{0}}\left[\left[\frac{1}{r_{0}^{2}}\right]\left[\frac{r^{3}}{3 G m_{1} m_{2}}\right]+\frac{G m_{1} m_{2}}{r}\right]\right]^{-1}\right]^{2}\left[\frac{\mathrm{d} r^{2}}{1-k r^{2}}+r^{2} \mathrm{~d} \theta^{2}+r^{2} \sin ^{2} \theta \mathrm{d} \phi^{2}\right]
\end{aligned}
$$

This establishes the IGIA relationship to the Friedman-Walker-Robertson metric, where constant $k$ in Equation (2.14) is equal to $+1(k=+1)$ and $r>r_{0}$ for positive spherical curvature describing the expansion of the cosmological fluid [3]. 
The inverse gravity inflationary assertion (IGIA) can be defined in terms of field theory via its relationship to the Klein-Gordon lagrangian. In formulating the expressions describing this relationship, IGIA potential energy $U_{T}(r)$ is set equal relativistic energy denoted $E_{\text {rel }}$ as shown below [1].

$$
U_{T}(r)=\left[p^{2} c^{4}+m^{2} c^{4}\right]^{1 / 2} \equiv E_{r e l}
$$

This can be expressed such that:

$$
E_{\text {rel }}=\left[\left[\frac{1}{r_{0}^{2}}\right]\left[\frac{r^{3}}{3 G m_{1} m_{2}}\right]+\frac{G m_{1} m_{2}}{r}\right]=\left[p^{2} c^{4}+m^{2} c^{4}\right]^{1 / 2}
$$

where $\phi\left(x_{\mathrm{L}}\right)$ is a scalar field function at Minkowski coordinates $x_{\mathrm{L}}$, the momenta $p$ is expressed as a tangent vector on the Minkowski coordinates $x_{ц}$ which is a function of (or parameterized by) time $t\left(x_{ц}=x_{ц}(t)\right)$ as shown below [3].

$$
p=\frac{1}{c} \frac{\partial \phi\left(x_{\amalg}\right)}{\partial t}=\frac{1}{c} \frac{\partial \phi\left(x_{\amalg}\right)}{\partial x_{\amalg}} \frac{\partial x_{\amalg}}{\partial t}=\frac{1}{c} \nabla_{ц} \phi\left(x_{\amalg}\right)
$$

Expressing Equation (2.16) in terms of field $\phi\left(x_{ц}\right)$ and substituting the value of momentum $p$ (of Equation (2.17)) into Equation (2.16) gives [5]:

$$
E_{r e l}\left(x_{\text {ц }}\right) \equiv\left[\left[\frac{1}{r_{0}^{2}}\right]\left[\frac{r^{3}}{3 G m_{1} m_{2}}\right]+\frac{G m_{1} m_{2}}{r}\right]=\left[\left(\nabla_{ц} \phi\left(x_{\text {ц }}\right)\right)^{2}+\left(m^{2}\left(\phi\left(x_{\text {ц }}\right)\right)^{2} c^{4}\right)\right]^{1 / 2}
$$

where the relativistic energy $E_{r e l}$ is expressed as a function of Minkowski coordinates $x_{ц}\left(E_{r e l}\left(x_{ц}\right)\right)$ which gives a form of the Klein-Gordon equation. The speed of light $c$ is set equal to unity $(c=1)$. A priori is that the differential momentum value $\nabla_{ц} \phi\left(x_{ц}\right)$ relates to the energy value of the IGIA such that (or solving Equation (2.18) for the differential term $\left.\nabla_{\mathrm{L}} \phi\left(x_{\mathrm{L}}\right)\right)$ :

$$
\nabla_{\mathrm{L}} \phi\left(x_{\mathrm{L}}\right)=\left[\left(E_{r e l}\left(x_{\mathrm{L}}\right)\right)^{2}-\left(m^{2}\left(\phi\left(x_{\mathrm{L}}\right)\right)^{2}\right)\right]^{1 / 2}
$$

Equation (2.19) can be alternatively expressed in terms of the IGIA such that:

$$
\nabla_{\text {ц }} \phi\left(x_{\text {ц }}\right)=\left[\left(\left[\left[\frac{1}{r_{0}^{2}}\right]\left[\frac{r^{3}}{3 G m_{1} m_{2}}\right]+\frac{G m_{1} m_{2}}{r}\right]\right)^{2}-\left(m^{2}\left(\phi\left(x_{ц}\right)\right)^{2}\right)\right]^{1 / 2}
$$

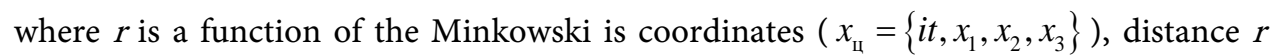
can be expressed such that [3]:

$$
r=\left[-t^{2}+\sum_{1}^{3}\left(x_{\mathrm{L}}\right)^{2}\right]^{1 / 2}=r\left(x_{\mathrm{L}}\right)
$$

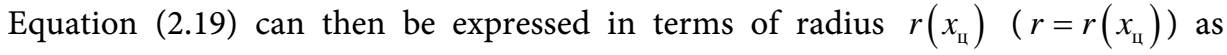
shown below.

$$
\nabla_{ц} \phi\left(x_{\amalg}\right)=\left[\left(\left[\left[\frac{1}{r_{0}^{2}}\right]\left[\frac{\left(r\left(x_{\amalg}\right)\right)^{3}}{3 G m_{1} m_{2}}\right]+\frac{G m_{1} m_{2}}{r\left(x_{\amalg}\right)}\right]\right)^{2}-m^{2}\left(\phi\left(x_{\amalg}\right)\right)^{2}\right]^{1 / 2}
$$


Therefore, we introduce momentum $p^{\prime}$ expressed as the differential term $\nabla^{\text {॥ }} \phi\left(x_{\text {u }}\right)$ (observe the superscript ц ) as shown below [3].

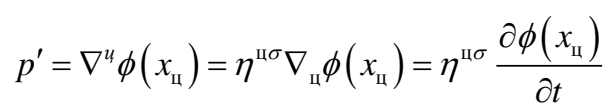

(Note: Recall thatthe speed of light $c$ is set equal to unity $(c=1))$ Where the Min-

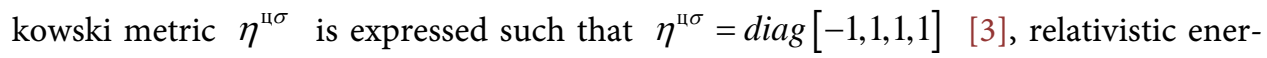
gy $E_{\text {rel }}^{\prime}\left(x_{\mathrm{u}}\right)$ corresponds to momentum $p^{\prime}$ and the IGIA such that:

$$
E_{\text {rel }}^{\prime}\left(x_{\text {џ }}\right) \equiv\left[\left[\frac{1}{r_{0}^{2}}\right]\left[\frac{r^{3}}{3 G m_{1} m_{2}}\right]+\frac{G m_{1} m_{2}}{r}\right]=\left[\left(\nabla^{\text {॥ }} \phi\left(x_{\text {щ }}\right)\right)^{2}+\left(m^{2} c^{4}\right)\right]^{1 / 2}
$$

Equation (2.25) below is the Klein-Gordon equation expressed such that [3]:

$$
\left(E_{K G} \phi\left(x_{\text {и }}\right)\right)^{2}=\nabla_{\text {ц }} \phi\left(x_{\text {и }}\right) \nabla^{\text {и }} \phi\left(x_{\text {ц }}\right)-m^{2}\left(\phi\left(x_{\text {ц }}\right)\right)^{2}
$$

Thus, as presented by Wald [3], the Klein-Gordon lagrangian is of the form shown below.

$$
L_{K G}=\frac{1}{2}\left(E_{K G} \phi\left(x_{\text {u }}\right)\right)^{2}=-\frac{1}{2}\left[\nabla_{\text {u }} \phi\left(x_{\text {u }}\right) \nabla^{u} \phi\left(x_{\text {u }}\right)+m^{2}\left(\phi\left(x_{\text {u }}\right)\right)^{2}\right]
$$

Expressing the Klein-Gordon lagrangian (Equation (2.26)) above in terms of the IGIA, the value of Equation (2.23) is substituted into Equation (2.26) (where $\left.\nabla^{\text {щ }} \phi\left(x_{\text {ц }}\right)=\eta^{\text {ц } \sigma} \nabla_{\text {ц }} \phi\left(x_{\text {ц }}\right)\right)$ giving:

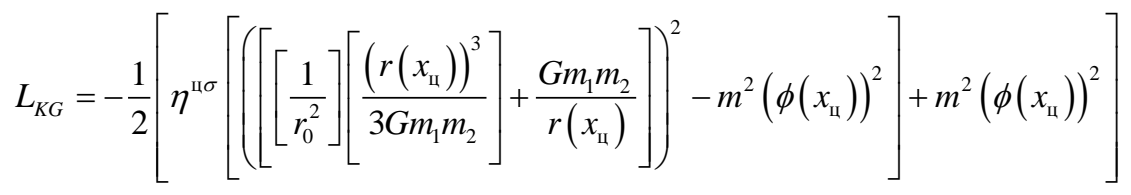

This implies that the product of differential terms $\nabla_{\text {ц }} \phi\left(x_{\text {u }}\right) \nabla^{\text {u}} \phi\left(x_{\mathrm{u}}\right)$ takes a value incorporating the IGIA such that:

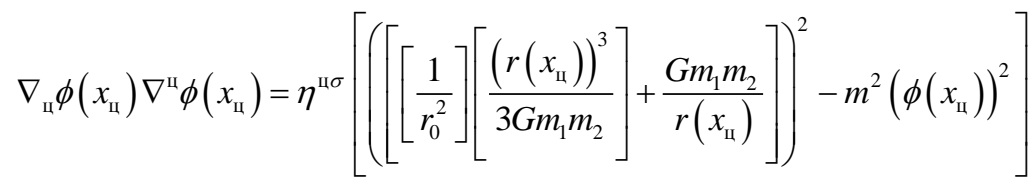

Solutions to Equations (2.27) and (2.28) pertain to mathematical methods of solving differential equations. Conclusively, Equation (2.28) is the IGIA correlation to various areas of field theory especially quantum energy fields describing vacuum energy (and the stress-energy tensor described in the next section). Lastly, dark energy $E_{\text {dark }}$ in terms of the IGIA is given by the conditions shown below.

$$
E_{\text {dark }}=\left[\frac{1}{r_{0}^{2}}\right]\left[\frac{r^{3}}{3 G m_{1} m_{2}}\right]
$$

Thus, dark energy is interpreted according to the IGIA as the inverse term of potential energy $U_{T}(r)$ (of Equation (2.01)). An important consideration is that the energy of expansion or energy $E_{\text {dark }}$ is different from the energy of the big bang which will be denoted energy $E_{B B}$ for the purposes of this explanation. Thus energy $E_{B B}$ is far 
greater in magnitude than the energy of gravity at the big bang denoted $U_{B B}=\left(G M m / r_{i}\right)$ (along with other elementary forces such as electromagnetism, the strong nuclear force, and the weak nuclear force opposing universal expansion) at the big bang where radius $r_{i}$ is the infinitesimally small distance of the big bang $\left(E_{B B}>U_{B B}\right)$. Where big bang energy $E_{B B}$ is composed of kinetic energy and electromagnetic energy of the universe, this implies that energy $E_{B B}$ is also sufficient for accelerating the total cosmological mass beyond the astronomical distance or radius $r_{0}$ to which cosmological expansion takes prominence over gravity and where energy $E_{\text {dark }}$ can generate cosmological expansion.

\section{The Dynamic Pressure of an Expanding Cosmological Fluid in Terms of the IGIT}

This section mathematically defines the dynamic pressure of a cosmological fluid in a homogeneous isotropic universe in terms of the IGIA. The stress-energy tensor for a perfect fluid used to describe the expansion of the cosmological fluid is given such that [3]:

$$
T_{a b}=\nabla_{a} \phi \nabla_{b} \phi+g_{a b} L_{K G}
$$

where $\phi$ is the field function of the space-time manifold [5] that the stress-energy tensor is defined on, $g_{a b}$ is the metric tensor, and $L_{K G}$ is the Klein-Gordon lagrangian such that [3]:

$$
L_{K G}=-\frac{1}{2}\left[\nabla^{c} \phi \nabla_{c} \phi+\phi^{2} m^{2} c^{2}\right]
$$

The 4 space tangent vectors $\nabla_{a} \phi \nabla_{b} \phi$ in Equation (3.0) obey the geodesic rule such that [3]:

$$
\nabla_{c}\left(\nabla_{a} \phi \nabla_{b} \phi\right)=\nabla_{c}\left(\nabla_{a} \phi \nabla_{b} \phi\right)+\Gamma_{a b}^{c}\left(\nabla_{a} \phi \nabla_{b} \phi\right) \equiv 0
$$

Thus showing the appropriate use of the Christoffle symbol $\Gamma_{a b}^{c}$ [3]. The partial derivative $\nabla_{c}$ in Equation (3.02) is of the form $\nabla_{c}=\left(\partial / \partial x_{c}\right)$. The tangent vector of the form $\nabla_{\mu} \phi$ is defined by the chain rule such that [2]:

$$
\nabla_{\mu} \phi=\frac{\partial \phi}{\partial x_{\Perp}} \frac{\partial x_{\Perp}}{\partial t}
$$

where the time coordinate has a value $c t$ and the speed of light $c$ is set to unity $(c=1)$ [5], the spatial coordinates $x_{\mathrm{u}}$ in $R^{4}$ are the Minkowski coordinates $\left(x_{\mathrm{u}}=\left\{i t, x_{1}, x_{2}, x_{3}\right\}\right.$ and $\left.x_{\mathrm{u}}=x_{\mathrm{u}}(t)\right)$ [5]. Thus the tangent vector $\nabla_{\mu} \phi$ at time $t$ is the 4 -velocity of the cosmological fluid denoted $u_{\text {ц }}\left(u_{\mathrm{u}} \in R^{4}\right)$ as shown below [3].

$$
u_{\mathrm{u}}=\nabla_{\mu} \phi=\frac{\partial \phi}{\partial x_{\mathrm{u}}} \frac{\partial x_{\mathrm{u}}}{\partial t}
$$

Therefore the product of tangent vectors $\nabla_{a} \phi$ and $\nabla_{b} \phi\left(\nabla_{a} \phi \nabla_{b} \phi\right)$ are given such that [3]:

$$
\nabla_{a} \phi \nabla_{b} \phi=u_{a} \cdot u_{b}=\sum_{\text {ц }}\left(u_{\text {и }}\right)^{2}
$$


It must be noted that tangent vectors $\nabla_{a} \phi$ and $\nabla_{b} \phi$ are symmetric $\left(\nabla_{a} \phi=\nabla_{b} \phi\right)$ which implies the fluid velocities $u_{a}$ and $u_{b}$ are also symmetric $\left(u_{a}=u_{b}=u_{\mathrm{u}}\right)$, therefore $u_{a} \cdot u_{b}=\sum_{ц}\left(u_{ц}\right)^{2}$. Total dynamic pressure $P_{a b}$ (where the subscripts $a b$ pertain to a 4 by 4 matrix) is given in terms of fluid 4 -velocity $u_{\mathrm{L}}$ such that [1] [3]:

$$
P_{a b}=\frac{\rho \sum_{\text {ц }}\left(u_{\text {ц }}\right)^{2}}{2}
$$

This implies that [1]:

$$
P_{a b}=\frac{\rho \sum_{\mathrm{u}}\left(u_{\mathrm{u}}\right)^{2}}{2}=\frac{\rho\left(\nabla_{a} \phi \nabla_{b} \phi\right)}{2}
$$

In the task of defining the expansion of the cosmological fluid in terms of the IGIT, consider the unit vector $\hat{u}$ in $R^{4}\left(\hat{u} \in R^{4}\right.$ and $\left.x_{\mu} \in R^{4}\right)$ shown below [2].

$$
\hat{u}=\frac{x_{\mu}}{\left|x_{\mu}\right|} \quad \text { such that } \hat{u} \cdot \hat{u}=1
$$

Multiplying unit vector $\hat{u}$ to the IGIT force value $F_{T}(r)$ of Equation (1.03) gives vector valued force $\left(F_{T}(r)\right)_{\mu}$ in $R^{4}\left(\left(F_{T}(r)\right)_{\mu} \in R^{4}\right)$ such that [2]:

$$
\left(F_{T}(r)\right)_{\mu}=\hat{u}\left(F_{T}(r)\right)
$$

This can be expressed such that:

$$
\left(F_{T}(r)\right)_{\mu}=\frac{x_{\mu}}{\left|x_{\mu}\right|}\left[F_{g}^{\prime}(r)-F_{g}(r)\right]
$$

where $A^{\prime} \quad\left(A^{\prime}=4 \pi r^{2}[3]\right)$ is a spherically symmetric area, the sum or superposition of 4 pressure components is as expressed below [3]. [Where pressure $=$ force $\div$ area $(P=F / A)][1]$

$$
\sum_{\mu} \frac{\left(F_{T}(r)\right)_{\mu}}{A^{\prime}}=\sum_{\mu}\left[\frac{x_{\mu}}{A^{\prime}\left|x_{\mu}\right|}\left[F_{g}^{\prime}(r)-F_{g}(r)\right]\right] \rightarrow \sum \frac{F}{A}
$$

(Note: that the pressure component $P_{00}$ of the 4 by 4 matrix of the stress-energy tensor has a value of energy density $\rho_{E}\left(P_{00}=\rho_{E}\right)$ [3] $)$ This is set equal to total dynamic pressure $P_{a b}$ of Equation (3.07) as shown below [1].

$$
P_{a b}=\frac{\rho \sum_{\text {ц }}\left(u_{\text {ц }}\right)^{2}}{2}=\sum_{\mu} \frac{\left(F_{T}(r)\right)_{\mu}}{A^{\prime}}
$$

This implies that [1]:

$$
\frac{\rho \sum_{ц}\left(u_{\text {ц }}\right)^{2}}{2}=\sum_{\mu} \frac{\left(F_{T}(r)\right)_{\mu}}{A^{\prime}}=\frac{\rho\left(\nabla_{a} \phi \nabla_{b} \phi\right)}{2} \equiv P_{a b}
$$

which also implies the equivalence of:

$$
\nabla_{a} \phi \nabla_{b} \phi=\frac{2}{\rho} \sum_{\mu} \frac{\left(F_{T}(r)\right)_{\mu}}{A^{\prime}} \equiv \frac{2}{\rho} P_{a b}
$$

Therefore this can be expressed such that: 


$$
\nabla_{a} \phi \nabla_{b} \phi=\frac{2}{\rho} \sum_{\mu}\left[\frac{x_{\mu}}{A^{\prime}\left|x_{\mu}\right|}\left[F_{g}^{\prime}(r)-F_{g}(r)\right]\right] \equiv \frac{2}{\rho} P_{a b}
$$

Now substituting the value of force term $F_{g}^{\prime}(r)-F_{g}(r)$ into Equation (3.15) gives:

$$
\nabla_{a} \phi \nabla_{b} \boldsymbol{\phi}=\frac{2}{\rho} \sum_{\mu}\left[\frac{x_{\mu}}{A^{\prime}\left|x_{\mu}\right|}\left[\left[\frac{1}{r_{0}^{2}}\right]\left[\frac{r^{2}}{G m_{1} m_{2}}\right]-\frac{G m_{1} m_{2}}{r^{2}}\right]\right] \equiv \frac{2}{\rho} P_{a b}
$$

Thence, substituting the value of $\nabla_{a} \phi \nabla_{b} \phi$ (shown above) for the value of Equation (3.15) into the stress-energy tensor $T_{a b}$, the stress-energy tensor can be expressed such that:

$$
T_{a b}=\frac{2}{\rho} \sum_{\mu}\left[\frac{x_{\mu}}{A^{\prime}\left|x_{\mu}\right|}\left[F_{g}^{\prime}(r)-F_{g}(r)\right]\right]-g_{a b} L_{K G}
$$

which is equivalent to the stress-energy tensor such that:

$$
T_{a b}=\frac{2}{\rho} \sum_{\mu}\left[\frac{x_{\mu}}{A^{\prime}\left|x_{\mu}\right|}\left[\left[\frac{1}{r_{0}^{2}}\right]\left[\frac{r^{2}}{G m_{1} m_{2}}\right]-\frac{G m_{1} m_{2}}{r^{2}}\right]\right]-g_{a b} L_{K G}
$$

Equation (3.18) can be expressed in matrix form such that:

$$
T_{a b}=\left[\begin{array}{cccc}
\rho_{E} & T_{01} & T_{02} & T_{03} \\
T_{10} & \left(\frac{2}{\rho} P_{11}-g_{11} L_{K G}\right) & T_{12} & T_{13} \\
T_{20} & T_{21} & \left(\frac{2}{\rho} P_{22}-g_{22} L_{K G}\right) & T_{23} \\
T_{30} & T_{31} & T_{32} & \left(\frac{2}{\rho} P_{33}-g_{33} L_{K G}\right)
\end{array}\right]
$$

The correlation of the stress-energy tensor $T_{a b}$ formulated in terms of the IGIA to Einstein's field equations describing an isotropic and homogeneous universe is given in the conclusion. Thus, we conclude with the formulation and incorporation of the IGIA in Einstein's field equation in its entirety.

\section{Conclusions: Einstein's Field Equations Describing an Expanding Homogeneous and Isotropic Universe in Terms of the IGIA}

In describing an expanding homogeneous isotropic universe in terms of the IGIA, it is of great importance that the IGIA is fully incorporated to Einstein's field equations as a whole. Thus, we began the heuristic derivation according to Wald [3] with expressions of Einstein's Field equations such that:

$$
\begin{aligned}
G_{t t} & =8 \pi T_{t t}=8 \pi \rho \\
G_{* *} & =8 \pi T_{* *}=8 \pi P
\end{aligned}
$$

The expressions of the stress-energy tensor in Equations (4.0) and (4.10 ( $T_{t t}$ and $\left.T_{* *}\right)$ are related the average value of cosmological mass density $\rho$ and to pressure value $P$ 
[3]. The two expressions of Einstein's tensor $G_{t t}$ and $G_{* *}$ are given such that [3]:

$$
\begin{aligned}
& G_{t t}=G_{a b} u^{a} u^{b} \\
& G_{* *}=G_{a b} s^{a} s^{b}
\end{aligned}
$$

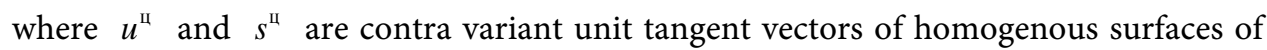
the isotropic and expanding universe [3]. Thus as depicted by Wald, the RobertsonWalker metric for describing a flat spatial geometry is expressed such that [3]:

$$
\mathrm{d} s^{2}=-\mathrm{d} t^{2}+a^{2}(t)\left(\mathrm{d} x^{2}+\mathrm{d} y^{2}+\mathrm{d} z^{2}\right)
$$

The scale factor at time $t$ denoted $a(t)$ in the metric above is equal to the value of scale factor $a\left(t_{e m}\right)$ of Equation (2.11) $\left(a(t)=a\left(t_{e m}\right)\right)$ in terms of the IGIA. This equivalence can be stated such that:

$$
a(t)=a\left(t_{e m}\right)=a_{0}\left[\frac{1}{E_{0}}\left[\left[\frac{1}{r_{0}^{2}}\right]\left[\frac{r^{3}}{3 G m_{1} m_{2}}\right]+\frac{G m_{1} m_{2}}{r}\right]\right]^{-1}
$$

Recall that for our purposes $t=t_{e m}$. Substituting the value of Equation (4.5) into Equation (4.4), the flat space Robertson-Walker metric of equation of (4.4) can be stated in terms of the IGIA (similarly to Robertson-Walker metric of Equation (2.14)) such that:

$$
\mathrm{d} s^{2}=-\mathrm{d} t^{2}+\left[a_{0}\left[\frac{1}{E_{0}}\left[\left[\frac{1}{r_{0}^{2}}\right]\left[\frac{r^{3}}{3 G m_{1} m_{2}}\right]+\frac{G m_{1} m_{2}}{r}\right]\right]^{-1}\right]^{2}\left(\mathrm{~d} x^{2}+\mathrm{d} y^{2}+\mathrm{d} z^{2}\right)
$$

Thus solving Equation (4.6) for the value of scale factor $a\left(t_{e m}\right)$ in Equation (4.6) gives the equivalence of values:

$$
a\left(t_{e m}\right)=\left[\left(\mathrm{d} s^{2}+\mathrm{d} t^{2}\right)\left(\mathrm{d} x^{2}+\mathrm{d} y^{2}+\mathrm{dz}^{2}\right)^{-1}\right]^{\frac{1}{2}}=a_{0}\left[\frac{1}{E_{0}}\left[\left[\frac{1}{r_{0}^{2}}\right]\left[\frac{r^{3}}{3 G m_{1} m_{2}}\right]+\frac{G m_{1} m_{2}}{r}\right]\right]^{-1}
$$

The time derivative of scale factor $a\left(t_{e m}\right)$ is denoted $\dot{a}$ (and $a$ is simply $a=a\left(t_{e m}\right)$ ) and can be expressed such that [3]:

$$
\dot{a}=\frac{\partial a\left(t_{e m}\right)}{\partial t}
$$

The coordinate $x_{\amalg}$ represent the Minkwoski coordinates $\left(x_{\amalg}=\{i t, x, y, z\}\right)[5]$. Where the time coordinate has a value of $c t$ and the speed of light $c$ is set to unity $(c=1)$ [5]. The question in reference to calculations is "how does the scale factor $a\left(t_{e m}\right)$ in terms of the IGIA (Equation (2.11) or (4.5)) relate to the time valued-derivative $\dot{a}$ of Equation (4.8) shown above?". The radius $r$ is defined on the Minkowski coordinates (it, $x, y, z$ ), thus the value (or magnitude) of radius $r$ (where $\left.r^{2}=\Delta s^{2}\right)$ is expressed such that [5]:

$$
\begin{aligned}
r^{2} & =\Delta s^{2}=\left[(\Delta t)^{2}+(\Delta x)^{2}+(\Delta y)^{2}+(\Delta z)^{2}\right]^{1 / 2} \\
& =\left[\left(i t-i t_{i}\right)^{2}+\left(x-x_{i}\right)^{2}+\left(y-y_{i}\right)^{2}+\left(z-z_{i}\right)^{2}\right]^{1 / 2}
\end{aligned}
$$


Distance $r$ is measured from the center of expansion (or the center of the universe), thus the initial values of $t_{i}, x_{i}, y_{i}$ and $z_{i}$ equal zero. Therefore substituting zero for all initial values $t_{i}, x_{i}, y_{i}$, and $z_{i}\left(\left(t_{i}, x_{i}, y_{i}, z_{i}\right)=(0,0,0,0)\right)$ and solving for radius $r$ in Equation (4.9) gives (Similarly to Equation (2.20)):

$$
r=\left[-t^{2}+x^{2}+y^{2}+z^{2}\right]^{1 / 2}
$$

Substituting the value of Equation (4.10) into Equation (2.11) (or Equation (4.5)) gives the IGIA scale factor as a function of the Minkowski coordinates $(a(t, x, y, z))$ such that:

$$
\begin{aligned}
a\left(t_{e m}\right) & =a(t, x, y, z) \\
& =a_{0}\left[\frac{1}{E_{0}}\left[\left[\frac{1}{r_{0}^{2}}\right]\left[\frac{\left(-t^{2}+x^{2}+y^{2}+z^{2}\right)^{3 / 2}}{3 G m_{1} m_{2}}\right]+\frac{G m_{1} m_{2}}{\left[-t^{2}+x^{2}+y^{2}+z^{2}\right]^{1 / 2}}\right]\right]^{-1}
\end{aligned}
$$

Thus pertaining to the time coordinate $\left(x_{0}=i t\right)$, the scale factor $a\left(t_{e m}\right)$ in terms of the IGIA is now differentiable to the time valued derivative of Equation (4.8), therefore permitting the continuation of the formulation without ambiguity. Equation (4.11) affords the opportunity to briefly present Hubble's constant in terms of the IGIA such that [3]:

$$
H(t)=\frac{\dot{a}}{a}=\left[\frac{1}{a(t, x, y, z)}\right] \frac{\mathrm{d} a(t, x, y, z)}{\mathrm{d} t}
$$

In continuing the IGIA's incorporation to Einstein's field equation, the scale factors a (keep in mind that $a=a(t)=a\left(t_{e m}\right)=a(t, x, y, z)$ ) relate to the symmetric Christoffel symbols such that [3]:

$$
\begin{aligned}
& \Gamma_{x x}^{t}=\Gamma_{y y}^{t}=\Gamma_{z z}^{t}=a \dot{a} \\
& \Gamma_{x t}^{x}=\Gamma_{t x}^{x}=\Gamma_{t y}^{y}=\Gamma_{y t}^{y}=\Gamma_{z t}^{z}=\Gamma_{t z}^{z}=\dot{a} / a
\end{aligned}
$$

Thus we acknowledge that the Christoffel symbols $\Gamma_{b c}^{a}$ is of the form [3]:

$$
\Gamma_{b c}^{a}=\frac{1}{2} \sum_{d} g^{a d}\left\{\frac{\partial g_{c b}}{\partial x^{b}}+\frac{\partial g_{c a}}{\partial x^{c}}-\frac{\partial g_{b c}}{\partial x^{c}}\right\}
$$

The components of the Ricci tensor are calculated according to the equation of [3]:

$$
R_{a b}=\sum_{c} R_{a c b^{c}}
$$

This can alternatively be expressed such that [3]:

$$
R_{a b}=\sum_{c} \frac{\partial y}{\partial x^{c}} \Gamma_{a b}^{c}-\frac{\partial}{\partial x^{a}}\left(\sum_{c} \Gamma_{c b}^{c}\right)+\sum_{d, c}\left(\Gamma_{a b}^{d} \Gamma_{d c}^{c}-\Gamma_{c b}^{d} \Gamma_{d a}^{c}\right)
$$

The Ricci tensor is then related to the scale factor $a$ (or $a\left(t_{e m}\right)$ in terms of the IGIA) by the equations of (where $\left.\ddot{a}=\mathrm{d}^{2}(a(t, x, y, z)) / \mathrm{d} t^{2}\right)[3]$ :

$$
\begin{aligned}
R_{t t} & =-3 \ddot{a} / a \\
R_{* * *} & =a^{-2} R_{x x}=\frac{\ddot{a}}{a}+2 \frac{(\dot{a})^{2}}{a^{2}}
\end{aligned}
$$


As stated by Wald, the value of Ricci tensor $R_{x x}$ in Equation (4.19) above relates to the Christoffel symbol such that [3]:

$$
R_{x x}=\sum_{c} \frac{\partial y}{\partial x^{c}} \Gamma_{x x}^{c}-\frac{\partial}{\partial x^{x}}\left(\sum_{c} \Gamma_{c x}^{c}\right)+\sum_{d, c}\left(\Gamma_{x x}^{d} \Gamma_{d c}^{c}-\Gamma_{c x}^{d} \Gamma_{d x}^{c}\right)
$$

Therefore the value of the scalar curvature $R$ is given such that [3]:

$$
R=-R_{t t}+3 R_{* * *}
$$

Substituting the value of Equation (4.18) and (4.19) into Equation (4.21) give a value such that [3]:

$$
R=-R_{t t}+3 R_{* *}=6\left(\frac{\ddot{a}}{a}+\frac{(\dot{a})^{2}}{a^{2}}\right)
$$

Conclusively, the values of Einstein tensor values $G_{t t}$ and $G_{* *}$ are given such that [3]:

$$
\begin{aligned}
& G_{t t}=R_{t t}+\frac{1}{2} R=\frac{3(\dot{a})^{2}}{a^{2}}=8 \pi \rho \\
& G_{* * *}=R_{* * *}-\frac{1}{2} R=-2 \frac{\ddot{a}}{a}-\frac{(\dot{a})^{2}}{a^{2}}=8 \pi P
\end{aligned}
$$

As stated by Wald, using Equation (4.23), Equation (4.24) can be rewritten such that [3]:

$$
\frac{3(\dot{a})^{2}}{a^{2}}=-4 \pi(\rho+3 P)
$$

Due to the fact that the description of the IGIA is defined in reference to a homogeneous and isotropic universe, the general evolutions for isotropic and homogeneous universe are given such that [3]:

$$
\begin{gathered}
\frac{3(\dot{a})^{2}}{a^{2}}=8 \pi \rho-\frac{3 k}{a^{2}} \\
\frac{3 \ddot{a}}{a}=--4 \pi(\rho+3 P)
\end{gathered}
$$

where scale factors $a$ and their corresponding time derivatives ( $\dot{a}$ and $\ddot{a}$ ) can be defined in terms of the IGIA of Equation (4.10) $(a=a(t, x, y, z))$, constant $k$ is equal to $+1(k=+1)$ and $r>r_{0}$ for positive spherical curvature describing the expansion of the cosmological fluid in an homogeneous isotropic universe [3]. At this juncture, the relationship of the scale factor of Equation (4.5) (and Equation (2.11)) in terms of the IGIA to Einstein's field equation have been formulated.

Equation (4.1) $\left(G_{* *}=8 \pi T_{* *}=8 \pi P\right)$ shows the relationship between pressure $P$ and the stress-energy tensor $T_{a b}$ of Equation 3.18 [3]. This implies that $P$ can be minimally substituted for the stress-energy tensor $\left(P \rightarrow T_{a b}\right)$. Thus, the pressure term $P$ relates the IGIA stress-energy tensor of Equation (3.18) (of the previous section) such that:

$$
P \rightarrow T_{a b}=\frac{2}{\rho} \sum_{\mu}\left[\frac{x_{\mu}}{A^{\prime}\left|x_{\mu}\right|}\left[\left[\frac{1}{r_{0}^{2}}\right]\left[\frac{r^{2}}{G m_{1} m_{2}}\right]-\frac{G m_{1} m_{2}}{r^{2}}\right]\right]-g_{a b} L_{K G}
$$


Resultantly, this can be expressed such that:

$$
P=\frac{2}{\rho} \sum_{\mu}\left[\frac{x_{\mu}}{A^{\prime}\left|x_{\mu}\right|}\left[\left[\frac{1}{r_{0}^{2}}\right]\left[\frac{r^{2}}{G m_{1} m_{2}}\right]-\frac{G m_{1} m_{2}}{r^{2}}\right]\right]-g_{a b} L_{K G}
$$

Spherically symmetric area $A^{\prime}$ is equal to a value of $8 \pi\left(A^{\prime}=8 \pi\right)$, therefore Equation (4.29) can be stated such that:

$$
P=\frac{2}{8 \pi \rho} \sum_{\mu}\left[\frac{x_{\mu}}{\left|x_{\mu}\right|}\left[\left[\frac{1}{r_{0}^{2}}\right]\left[\frac{r^{2}}{G m_{1} m_{2}}\right]-\frac{G m_{1} m_{2}}{r^{2}}\right]\right]-g_{a b} L_{K G}
$$

Substituting the value of pressure $P$ presented above into Equation (4.27) gives a value such that:

$$
\frac{3 \ddot{a}}{a}=-4 \pi\left(\rho+3\left[\frac{2}{8 \pi \rho} \sum_{\mu}\left[\frac{x_{\mu}}{\left|x_{\mu}\right|}\left[\left[\frac{1}{r_{0}^{2}}\right]\left[\frac{r^{2}}{G m_{1} m_{2}}\right]-\frac{G m_{1} m_{2}}{r^{2}}\right]\right]-g_{a b} L_{K G}\right]\right)
$$

Equation (4.31) gives an additional incorporation of the Mathematics of the IGIA showing that the theoretical concept is well ingrained to the cosmological structure of the universe. The incorporation of the IGIA mathematics to Einstein's field equations gives a complete description to validate the concept and convey a new theoretical possibility to the physics community.

\section{Acknowledgements}

I would like to thank Juanita Walker and Mr. Eugene Thompson for their encouragement and support to this endeavor.

\section{References}

[1] Young, H.D., Freedman, R.A. (2004) Sears and Zemansky's University Physics. 11th Edition, Addison Wesley, San Francisco, Cal., Pearson, 444, 445, 454-457, 517, 529, 530, 1431, 1450 .

[2] Stewart, J. (2003) Calculus. 5th Edition, Belmont, Cal., Thompson, Brooks/Cole, 877, 968, 1095.

[3] Wald, R.M. (1984) General Relativity. Chicago Press, Ltd., Chicago, 17, 23-26, 31-38, 59, 70, 72, 95, 96, 101-104, 120-124.

[4] Mukhanov, V.F. (2005) Physical Foundations of Cosmology. Cambridge University Press, Cambridge, New York, Page 58. http://dx.doi.org/10.1017/CBO9780511790553

[5] Penrose, R. (2004) The Road to Reality: Comprehensive Guide to the Laws of the Universe. Alfred A. Knopf Publishing, New York, 222-223, 412, 413. 
Submit or recommend next manuscript to SCIRP and we will provide best service for you:

Accepting pre-submission inquiries through Email, Facebook, LinkedIn, Twitter, etc. A wide selection of journals (inclusive of 9 subjects, more than 200 journals)

Providing 24-hour high-quality service

User-friendly online submission system

Fair and swift peer-review system

Efficient typesetting and proofreading procedure

Display of the result of downloads and visits, as well as the number of cited articles

Maximum dissemination of your research work

Submit your manuscript at: http://papersubmission.scirp.org/

Or contact jmp@scirp.org 